\title{
Novel association between Helicobacter pylori infection and gastrointestinal stromal tumors (GIST) in a multi-ethnic population
}

\author{
Jaclyn Kagihara ${ }^{1}$, Brent Matsuda ${ }^{1}$, Kraig L. Young ${ }^{1}$, Xiufen Li $^{1}$, Xuegang Lao ${ }^{1}$, Gautam A. Deshpande ${ }^{1,2}$, \\ Fumio Omata ${ }^{2,3}$, Terrilea Burnett ${ }^{4}$, Charles F. Lynch ${ }^{5}$, Brenda Y. Hernandez ${ }^{4,6}$, Scott K. Kuwada,4 \\ ${ }^{1}$ Department of Medicine, University of Hawaii John A. Burns School of Medicine, Honolulu, HI, USA; ${ }^{2}$ Department of General Internal Medicine, \\ St. Luke's International University, Tokyo Japan; ${ }^{3}$ Department of Gastroenterology Division, St. Luke's International University, Tokyo Japan; \\ ${ }^{4}$ University of Hawaii Cancer Center, University of Hawaii, Honolulu, HI, USA; ${ }^{5}$ Department of Epidemiology, University of Iowa, Iowa City, IA, \\ USA; ${ }^{6}$ Hawai'i $i$ Tumor Registry, University of Hawaii Cancer Center, University of Hawaii, Honolulu, HI, USA \\ Contributions: (I) Conception and design: SK Kuwada; (II) Administrative support: SK Kuwada; (III) Provision of study materials or patients: \\ BY Hernandez, T Burnett, CF Lynch; (IV) Collection and assembly of data: SK Kuwada, BY Hernandez, X Li, X Lao; (V) Data analysis and \\ interpretation: SK Kuwada, J Kagihara, B Matsuda, KL Young, BY Hernandez, F Omata, GA Deshpande; (VI) Manuscript writing: All authors; (VII) \\ Final approval of manuscript: All authors. \\ Correspondence to: Scott K. Kuwada, MD. Professor of Medicine and Chief of Gastroenterology, University of Hawaii John A. Burns School of \\ Medicine, 550 S. Beretania St., Suite 501, Honolulu, HI 96813, USA. Email: skkuwada@hawaii.edu.
}

Background: The incidence of gastrointestinal stromal tumors (GIST) is increasing though its epidemiology remains poorly understood. The epidemiological factors involved in GIST were examined in the multi-ethnic population of Hawaii, which has the highest incidence of GIST of all Surveillance, Epidemiology and End Results (SEER) sites in the USA, in order to gain insight into the potential risk factors for GIST.

Methods: Archival tumor tissue from 71 morphologically and immunohistochemically confirmed GIST cases and 65 gastritis-only controls diagnosed between 1998-2017 were evaluated for five Helicobacter pylori (H. pylori) genes (HP1177, $16 \mathrm{~S} r R N A$, ice A, ureB, vacA) by polymerase chain reaction (PCR) and gastritis controls.

Results: Across the ethnically diverse Hawaii population, GIST were significantly more common in Asians compared with whites. The gastric predominance of GIST and higher prevalence of $H$. pylori in Asians than whites in Hawaii led us to examine this infection as a potential causative factor of GIST. Forty-nine $(69.0 \%)$ GIST cases were gastric in origin. Of 71 GIST cases, 48 (67.6\%) were positive for at least one H. pylori gene, compared with only 13 (20.0\%) of the controls \{unadjusted odds ratio (OR): 8.3 [95\% confidence interval (CI): 3.8-18.3]; $\mathrm{P}<0.0001$; 23 (32.4\%) of GIST cases were positive for at least two different $H$. pylori genes, compared with only 6 (9.2\%) controls [unadjusted OR: 4.7 (95\% CI: 1.8-12.5); P=0.002].

Conclusions: $H$. pylori infection is strongly associated with GIST and may play an important role in its tumorigenesis.

Keywords: Gastrointestinal stromal tumors (GIST); Helicobacter pylori (H. pylori); gastric cancer

Received: 17 February 2020; Accepted: 25 September 2020; Published: 10 November 2020.

doi: 10.21037 /gist-20-2

View this article at: http://dx.doi.org/10.21037/gist-20-2 


\section{Introduction}

Gastrointestinal stromal tumors (GIST) are relatively rare tumors affecting approximately 5,000 newly diagnosed patients annually in the USA, but are the most common mesenchymal tumors of the gastrointestinal tract. GIST were called leiomyomas and leiomyosarcomas in the past, but recent studies have identified interstitial cells of Cajal as the cell of origin of GIST, distinguishing GIST from other mesenchymal GI tumors (1-3). c-KIT (CD117) is expressed in $80-100 \%$ and CD34 in $50-80 \%$ of GIST, respectively $(1,4)$, and their expression is used to help diagnose GIST. DOG-1 and $\mathrm{PKC}_{\theta}$ are commonly expressed by GIST as well and are particularly useful in the identification of c-KIT-negative stromal tumors (5-9). Previous reports have identified c-KIT and PDGFRA mutations in the vast majority of GIST $(1,2)$.

Although there are multiple studies that examine the histology, pathogenesis, and treatment of GIST, existing data regarding the epidemiology of GIST are limited. Few publications to date have addressed potential ethnic differences in the incidence or prevalence of GIST. A population-based study of 292 GIST patients and 292 ageand sex-matched controls from the U.S. National Cancer Institute's Surveillance, Epidemiology and End Results (SEER) registry database, found no statistically significant differences in geographic region, location of residence, or median income, but found significantly more white controls than cases compared with blacks and other nonwhites (10). Another retrospective SEER study examined 1,458 malignant GIST cases from 1992 to 2000 in 12 SEER registry regions and found the highest incidence rate in Hawaii $(1.06 / 100,000$ person-years compared to the national age-adjusted incidence rate of $0.68 / 100,000$ person-years) (11). This led us to examine the factors associated with Hawaii having the highest incidence of GIST among SEER regions in the USA.

We present the following article in accordance with the MDAR reporting checklist (available at http://dx.doi. org/10.21037/gist-20-2).

\section{Methods}

\section{Epidemiology of GIST}

The Hawaii SEER registry data were queried and all diagnoses of malignant gastrointestinal stromal sarcoma (ICD-O-3 code 8936) from 1998 to 2009 were identified. The Hawaii Tumor Registry (HTR) has been part of the
U.S. SEER program since 1973.

Diagnosis of GIST was based on morphology (spindle, epithelioid, mixed) and immunohistochemical tumor markers (c-KIT, DOG1 or PDGFRA). Prior to 1998 , the terms leiomyoma, leiomyosarcoma, neurofibromas, schwannomas were more commonly used to refer to GIST, however because of their location and lack of immunohistochemical staining, were largely indistinguishable from GIST. To avoid historical coding "bias", sampling bias, or classification errors, tumors with these diagnoses (leiomyoma, leiomyosarcoma, neurofibroma, schwannoma) prior to 1998 were excluded from our data set. The HTR database includes patient demographics, tumor histology, primary site topography, tumor behavior, grade, and stage of disease at diagnosis, and immunohistochemistry in more recent cases.

The U.S. Census data (year 2000) was used to determine the ethnic composition of Hawaii's population as a reference for the ethnicity of GIST cases (12). Pacific Islander is a term that includes Chamorro, Micronesian, Marshallese, Samoan, and Tongan ethnic groups. Ethnicity was selfreported in both the SEER and U.S. Census. The incidence rates for GIST were compared amongst ethnic groups reported to the HTR from 1998-2009. The incidence rates in non-white ethnic groups were compared to the incidence rate in whites.

\section{Histological specimens}

The Residual Tissue Repositories of two SEER registries in Hawaii and Iowa were queried for de-identified malignant GIST tissue cases (ICD-O-3 code 8936) cases from 19982017. Formalin-fixed and paraffin-embedded (FFPE) GIST tissue sections (cases; $n=63$ ) were collected from the HTR and Queen's Medical Center. An additional eight malignant GIST cases from 2000-2015 were provided by the State Health Registry of Iowa due to the smaller number of whites with GIST in Hawaii. All GIST surgical resection specimens contained tumor bordered by non-malignant gastrointestinal tissue.

The controls were chosen from diagnostic esophagogastroduodenoscopy cases performed at the Queen's Medical Center from 2015-2017 found to have gastritis without evidence of tumors. Multiple (4-8) pinch biopsy specimens from the gastric corpus and antrum were obtained from 65 control patients who were referred for evaluation of upper gastrointestinal symptoms or anemia, and were found to have endoscopic and histopathologic 
evidence of gastritis without evidence of gastric malignancies or peptic ulcer disease.

\section{DNA extraction and purification}

For cases and controls, sequential sections ( $5 \mu \mathrm{m}$ thick) were cut from the tissue samples and mounted on glass microscope slides. For cases, one of the slices was stained with hematoxylin and eosin to verify the presence of tumor surrounded by adjacent normal gastrointestinal tissue. For controls, one of the slices was stained with hematoxylin and eosin to verify the presence of gastric tissue. The tissue specimens were scraped from 2-3 sequential unstained slides for each case and control. Both tumor and normal surrounding gastrointestinal tissue was collected for the GIST cases. The DNA was extracted from the paraffinembedded tissue sections as previously described (13). Briefly, paraffin-embedded sections were placed in $180 \mu \mathrm{L}$ ATL lysis buffer from the DNAeasy kit (Qiagen, Germany), followed by incubation at $100{ }^{\circ} \mathrm{C}$ for 30 minutes. The tubes were briefly centrifuged to remove condensate. Twenty $\mu \mathrm{L}$ of proteinase $\mathrm{K}$ was added to the content of the microfuge tubes, and the tubes were incubated at $65^{\circ} \mathrm{C}$ for 16 hours. The lysed emulsion was further purified with the DNAeasy spin-column (Qiagen). DNA was finally recovered in a single elution step with $100 \mu \mathrm{L} \mathrm{AE}$ solution from the DNAeasy kit (Qiagen).

\section{Detection of Helicobacter pylori (H. pylori)}

The following polymerase chain reaction (PCR) primer pairs were used to perform PCR amplification of $H$. pylorispecific genes from tumor DNA:

16S rRNA: 109 bp (14)

F: 5'-CTGGAGAGACTAAGCCCTCC-3'

R: 5'-ATTACTGACGCTGATTGTGC-3'

HP1177: 187 bp (14)

F: 5'-ACGAACGCGCAAAAACTTTA-3'

R: 5'-TTGCCATTCTCATCGGTGTA-3'

vacA: 136/163 bp (14)

F: 5'-ATGGAAATACAACAAACACAC-3'

R: 5'-CAACAATGGCTGGAATGAT-3'

ureB: 132 bp (15)

F: 5'-CCCATTTGACTCAATGCGATG-3'

R: 5'-TGGGATTAGCGAGTATGTCGG-3'

iceA1: 218 bp (16)

F: 5'-ATCATAAAGACGGCCGCAAAGAT-3'

R: 5'-AT(A/G)GGGTCATATTGATAACA(A/G)CC-3'
iceA2: 247 bp (16)

F: 5'-CGCTGTTTTTCTAGCGGTGTTTTA-3'

R: 5'-CATTGATCT(A/G)TGTTTGTATGCTTC-3'

(ice $A 1$ and ice $A 2$ are allelic variants of iceA. Detection of either allele was considered positive for iceA).

Human beta-globulin was used as a PCR control for the extraction of human DNA from the tissues:

$b g l$ [beta-globulin (control)]: 110 bp (17)

F: 5'-ACACAACTGTGTTCACTAGC-3'

R: 5'-CAACTTCATCCACGTTCACC-3'

PCR was performed on purified tumor DNA in $20 \mu \mathrm{L}$ goTaq green PCR buffer (PROMEGA, USA) containing forward and reverse primers. PCR was performed using a GeneAmp PCR System 9700 (Applied Biosystems, USA) with the following conditions: 6 minutes of preincubation at $94^{\circ} \mathrm{C}$ followed by 40 cycles of 30 seconds each at $94^{\circ} \mathrm{C}$, 30 seconds at $55^{\circ} \mathrm{C}$ and 30 seconds at $72^{\circ} \mathrm{C}$. Final extension was performed for 7 minutes at $72{ }^{\circ} \mathrm{C}$. The PCR products were resolved on $2 \%$ agarose gels, and, the respective bands were cut out of the agarose gels followed by DNA isolation and purification. Sequencing of the PCR products extracted from the gels was performed to verify the PCR products. The purified PCR products were sequenced using the same primers above and the resulting sequences matched using Nucleotide BLAST with matches yielding significance at $\mathrm{P}<0.05$. Both negative and positive (patients with positive $H$. pylori infection by Giemsa stain of gastric biopsies) controls were utilized for all PCR assays. The uniformity of DNA quality was determined by detection of the housekeeping gene $b g l$ by PCR. The PCR detection of the H. pylorispecific genes was performed in duplicate for each sample.

Approval for this research was granted by the Western Institutional Review Board (protocol no. 1168906) and University of Hawaii Human Subjects Committee (2014-302). This study conformed to the provisions of the Declaration of Helsinki (as revised in 2013). Due to the retrospective nature of the data and biospecimen collection, and de-identification of all personal health information, individual consent for this retrospective analysis was waived.

\section{Statistical analysis}

In addition to descriptive statistical analyses, bivariate analyses were performed to compare data; chi-square tests were used to determine the statistical significance between the frequency of GIST in various minority ethnic groups. Crude and unconditional logistic regression analyses were performed using SPSS v22 (IBM Corp., Armonk, NY, 
Table 1 Characteristics of GIST in the Hawaii SEER database from 1998-2009

\begin{tabular}{|c|c|c|}
\hline Characteristics & Total & Percentage \\
\hline \multicolumn{3}{|l|}{ Age at diagnosis, yr } \\
\hline$<50$ & 26 & 17.6 \\
\hline $50-59$ & 36 & 24.5 \\
\hline $60-69$ & 32 & 29.2 \\
\hline $70-79$ & 33 & 29.9 \\
\hline$>80$ & 20 & 13.6 \\
\hline \multicolumn{3}{|l|}{ Gender } \\
\hline Male & 73 & 49.7 \\
\hline Female & 74 & 50.3 \\
\hline \multicolumn{3}{|l|}{ Location } \\
\hline Stomach & 82 & 56.0 \\
\hline Small intestine & 48 & 34.0 \\
\hline Colon & 5 & 3.5 \\
\hline Other digestive organ & 11 & 7.5 \\
\hline \multicolumn{3}{|l|}{ Stage } \\
\hline Localized & 80 & 54.4 \\
\hline Regional & 26 & 17.7 \\
\hline Distant & 33 & 22.4 \\
\hline Unstaged & 8 & 5.4 \\
\hline
\end{tabular}

GIST, gastrointestinal stromal tumors; SEER, Surveillance, Epidemiology and End Results.

Table 2 Analysis of GIST cases by ethnicity compared to whites in Hawaii SEER Database from 1998-2009

\begin{tabular}{lc}
\hline Ethnicity & $P$ value \\
\hline Chinese & $4.7 \times 10^{-5}$ \\
Filipino & 0.03 \\
Hawaiian/part-Hawaiian & 0.2 \\
Japanese & $4.3 \times 10^{-4}$ \\
Korean & $1.5 \times 10^{-7}$ \\
Vietnamese & $2.6 \times 10^{-9}$ \\
White & 1 \\
Pacific Islander & $3.3 \times 10^{-7}$ \\
\hline
\end{tabular}

GIST, gastrointestinal stromal tumors; SEER, Surveillance, Epidemiology and End Results.
USA) to calculate risk [odds ratio (OR); 95\% confidence interval (CI)] of detection of $H$. pylori genes in all 71 GIST cases (from Hawaii and Iowa) versus the 66 controls (from Hawaii). All 95\% CI were two-sided. $\mathrm{P}<0.05$ was considered statistically significant.

\section{Results}

One hundred and forty-seven patients with a confirmed histologic diagnosis of malignant GIST were reported in the Hawaii SEER database from 1998-2009 (Table 1). Fifty-six percent of GIST were in the stomach, $34.0 \%$ in the small intestine, and $3.5 \%$ in the colon (Table 1). There was no significant difference in the prevalence of GIST between men or women of any ethnic group (Table 1). Fiftyfour point four percent of GIST were staged as localized, $17.7 \%$ regional, $22.4 \%$ with distant metastases, and $5.4 \%$ unstaged at the time of diagnosis (Table 1). Ethnicity was reported for all individuals except for $0.7 \%$ (no ethnicity reported) of GIST subjects. Compared to whites, malignant GIST incident rates were significantly greater in Japanese $\left(\mathrm{P}=4.3 \times 10^{-4}\right)$, Chinese $\left(\mathrm{P}=4.7 \times 10^{-5}\right)$, Korean $\left(\mathrm{P}=1.5 \times 10^{-7}\right)$, Vietnamese $\left(\mathrm{P}=2.6 \times 10^{-9}\right)$, Pacific Islander $\left(\mathrm{P}=3.3 \times 10^{-7}\right)$, and Filipino $(\mathrm{P}=0.03)$ ethnic groups in Hawaii (Table 2). The gastric and Asian predominance of GIST in the multiethnic Hawaii population was reminiscent of a previous study that strongly linked $H$. pylori infection to gastric adenocarcinoma in Hawaii Asians (18). Multiple studies have found a high coincidence of GIST and non-GIST cancers (mostly adenocarcinomas) in the stomachs and gastroesophageal junctions of the same patients, suggesting common risk factors for the two types of tumors (19-23). These data led us to look for an association between $H$. pylori infection and GIST.

Paraffin-embedded GIST tumor specimens were available for 71 GIST and 65 gastritis controls. Of the GIST cases, $11(15.5 \%)$ ranged in age 21 to 49 years, 13 (18.3\%) were 50 to 59 years, $14(19.7 \%)$ were 60 to 69 years, 21 $(29.6 \%)$ were 70 to 79 years, and $12(16.9 \%)$ were $>80$ years (Table 3). Of the controls, $16(24.6 \%)$ ranged in age 21 to 49 years, $11(16.9 \%)$ were 50 to 59 years, $19(29.2 \%)$ were 60 to 69 years, $12(18.5 \%)$ were 70 to 79 years, and $7(10.8 \%)$ were $>80$ years (Table 3). Females comprised $49.3 \%$ and $60 \%$ of GIST patients and controls, respectively (Table 3). Asians made up the majority of both GIST and controls at $42(59.2 \%)$ and $43(66.2 \%)(\mathrm{P}=0.40)$, 
Table 3 Demographic characteristics of GIST cases and controls for which tissue samples were collected

\begin{tabular}{|c|c|c|c|c|}
\hline Characteristics & \multicolumn{3}{|c|}{ GIST } & Controls $(n=65)$ \\
\hline \multicolumn{5}{|l|}{ Age at diagnosis, yr, $\mathrm{n}(\%)$} \\
\hline$<50$ & $10(15.9)$ & $1(12.5)$ & $11(15.5)$ & $16(24.6)$ \\
\hline $50-59$ & $13(20.6)$ & $0(0.0)$ & $13(18.3)$ & $11(16.9)$ \\
\hline $70-79$ & $17(27.0)$ & $4(50.0)$ & $21(29.6)$ & $12(18.5)$ \\
\hline$>80$ & 9 (14.3) & $3(37.5)$ & $12(16.9)$ & $7(10.8)$ \\
\hline \multicolumn{5}{|l|}{ Gender, n (\%) } \\
\hline Male & $32(50.8)$ & $4(50.0)$ & $36(50.7)$ & $26(40.0)$ \\
\hline \multicolumn{5}{|l|}{ Location, n (\%) } \\
\hline Stomach & $41(65.1)$ & $8(100.0)$ & $49(69.01)$ & $65(100.0)$ \\
\hline Small intestine & $16(25.4)$ & $0(0.0)$ & $16(22.54)$ & - \\
\hline Colon & $2(3.2)$ & $0(0.0)$ & $2(2.82)$ & - \\
\hline Other digestive organ & $4(6.3)$ & $0(0.0)$ & $4(5.63)$ & - \\
\hline
\end{tabular}

GIST, gastrointestinal stromal tumors.

respectively (Table S1). The remainder of GIST and controls were comprised of Hawaiians $9(12.7 \%)$ and 5 (7.7\%) $(\mathrm{P}=0.34)$, whites $14(19.7 \%)$ and $13(20.0 \%)$ $(\mathrm{P}=0.97)$, Pacific Islanders $5(7.0 \%)$ and $4(6.2 \%)(\mathrm{P}=0.85)$, and "other" (ethnicity not reported) $1(1.4 \%)$ and $0(0.0 \%)$ $(\mathrm{P}=0.34)$, respectively (Table S1). Of the primary GIST, $49(69.0 \%)$ were gastric in origin, $16(22.5 \%)$ occurred in the small intestines, $2(2.8 \%)$ in the colon, $2(2.8 \%)$ in the rectum and $2(2.8 \%)$ were detected outside the stomach and intestines (liver, mesentery) (Table 3). Among small intestine GIST ( $\mathrm{n}=16), 5$ were duodenal (31.3\%), 3 ileal $(18.8 \%), 1$ overlapped different segments of small intestine $(6.3 \%)$, and 7 were not otherwise specified (43.8\%).

H. pylori infection was detected by positive PCR amplification of five $H$. pylori genes (HP1177, $16 \mathrm{~S} r R N A$, $i c e A$, ure $B$, vacA1). For individual $H$. pylori genes, HP1177 was detected in 21 GIST and 1 control $(\mathrm{P}<0.0001), 16 S$ $r R N A$ in 18 GIST and 2 controls $(\mathrm{P}=0.0003)$, ice $A$ in 25 GIST and 9 controls $(\mathrm{P}=0.0041)$, ure $B$ in 15 GIST and 5 controls $(\mathrm{P}=0.028)$, and vacA in 14 GIST and 4 controls $(\mathrm{P}=0.021)$ (Table S2). Of the total 71 patients with GIST, $48(67.6 \%)$ were positive for at least one $H$. pylori gene, compared with only 13 (20.0\%) of 65 controls [unadjusted
OR: 8.3 (95\% CI: 3.8-18.3); $\mathrm{P}<0.0001]$ (Table 4). Furthermore, $23(32.4 \%)$ cases were positive for at least two $H$. pylori genes, compared with only $6(9.2 \%)$ controls [unadjusted OR: 4.7 (95\% CI: 1.8-12.5); $\mathrm{P}=0.002$ ] (Table 4). For the eight GIST cases from Iowa, 5 (62.5\%) were positive for at least one $H$. pylori gene (Table 3). Excluding the eight patients from Iowa did not alter the significance between GIST and $H$. pylori genes compared with controls.

Of the GIST cases that were positive for one or more H. pylori genes ( $\mathrm{n}=48), 32(66.7 \%)$ were gastric, $11(22.9 \%)$ were found in the small intestines, $1(2.1 \%)$ in the colon, $2(4.2 \%)$ occurred in the rectum, and $2(4.2 \%)$ were extraintestinal (liver, mesentery) (Table S1). On further analysis of the 11 small intestine GIST cases positive for one or more H. pylori genes, 4 (36.4\%) were duodenal, 3 ileal (27.3\%), 3 (27.3\%) overlapping small intestinal segments and $1(9.1 \%)$ not otherwise specified.

\section{Discussion}

In our study, we found GIST cases in Hawaii were overrepresented in Asians and Pacific Islanders compared 
Table 4 Summary of H. pylori gene detection in gastrointestinal tissues of GIST cases and controls

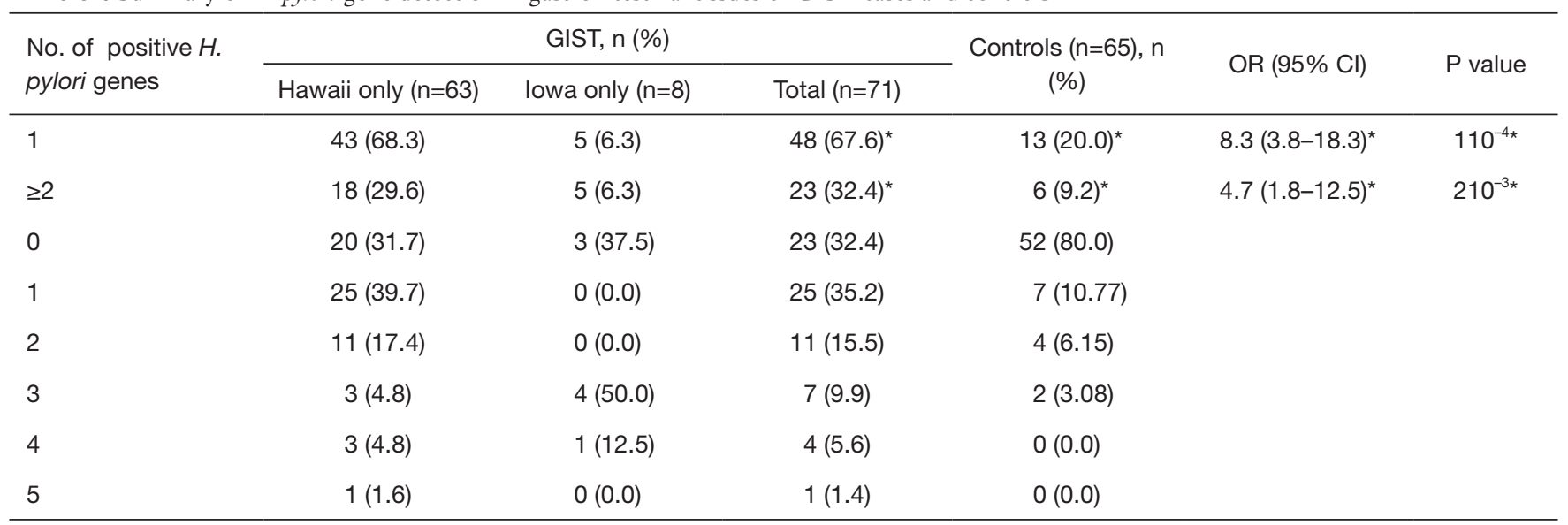

*, P<0.05 for GIST vs. controls. H. pylori, Helicobacter pylori; GIST, gastrointestinal stromal tumors; OR, odds ratio; Cl, confidence interval.

with whites. Recent studies found a higher prevalence of $H$. pylori infection in non-whites than whites in Hawaii (24), which may help explain the higher incidence rates for GIST in Asians and Pacific Islanders than whites in our study.

Previous studies demonstrated strong associations between $H$. pylori infection and two other types of gastric cancers: adenocarcinoma (18) and MALT lymphoma (25). The association between $H$. pylori infection and gastric adenocarcinoma led the International Agency for Research on Cancers to declare H. pylori a carcinogen (26). One study, published only in abstract form, did report an association between GIST and H. pylori infection (27). In this study, of 71 malignant GIST cases and 65 nonGIST controls, gastrointestinal specimens were positive for $H$. pylori infection by Giemsa stain in $62.9 \%$ and $30 \%$, respectively $(\mathrm{P}=0.013)$. Our study utilized $\mathrm{PCR}$ detection of $H$. pylori infection in gastrointestinal tissue specimens since this has been shown to be more sensitive than histochemical methods for $H$. pylori infection $(28,29)$ and allows unequivocal distinction between various Helicobacter species, such as $H$. helmanii, that can also reside in the stomach (30). In the regression analyses performed on our subjects, GIST cases showed large ORs for $H$. pylori infection detected by PCR compared with controls. Due to the much lower number of GIST cases in whites than Asians in Hawaii, we obtained 8 GIST specimens from eight white patients from the University of Iowa to determine if this could be due to sampling error. The majority of the GIST cases from Iowa were positive for $H$. pylori infection as well. Even when the subset of 63 GIST cases and 66 matched (age, gender, ethnicity) controls from Hawaii were analyzed separately, the ORs for the association between $H$. pylori and GIST remained large and significant. Thus, our results strongly suggest an association between gastrointestinal $H$. pylori infection and GIST.

With regards to the controls, we selected patients referred for evaluation of upper gastrointestinal symptoms or anemia, who were found to have endoscopic and histopathologic evidence of gastritis without evidence of gastric malignancies or peptic ulcer disease after careful consideration. We did consider patients with known peptic ulcer disease as controls but previous studies have shown an inverse correlation between duodenal ulcers and gastric cancer, and a direct correlation between gastric ulcers and gastric cancer (31-34). Thus, including duodenal ulcer patients as controls would have potentially skewed the ORs for $\mathrm{H}$. pylori infection in favor of GIST cases. Furthermore, using patients with gastric ulcers as controls would possibly have diminished the likelihood ratios for $H$. pylori infection and GIST, but may have included patients at risk for both gastric ulcers and GIST.

In our study, $H$. pylori genes were detected not just in gastric GIST but in small intestinal, colonic, and extraintestinal GIST as well. This is not unexpected since $H$. pylori has been detected in the small and large intestines of patients with gastrointestinal diseases and even in the liver of patients with liver cancers (35-37). Nevertheless, the majority of primary GIST tumors in Hawaii occur in the stomach, where $H$. pylori infection is most prevalent. Gastric adenocarcinoma is much more frequent than gastric MALT lymphoma and GIST, likely due to the fact that the latter malignancies originate in the submucosal layers of the 
stomach which underlie the epithelium and therefore are more distant from $H$. pylori and carcinogens.

In the USA, there is no recommendation for gastric cancer screening in the general population due to the relatively low overall rate of gastric cancer. However, clinicians should be aware of the increased frequency of GIST, in addition to gastric adenocarcinoma and MALT lymphoma, in patients at increased risk for or with $H$. pylori infection. As GIST arise within the gastrointestinal submucosa, they first appear as raised lesions covered by normal appearing mucosa and frequently require endoscopic ultrasound with fine needle aspiration for further characterization (38). Future studies should determine if eradication of $H$. pylori infection decreases the rate of GIST.

\section{Acknowledgments}

Funding: Centers of Biomedical Research Excellence Program (award P20 RR018727 from the National Center for Research Resources, National Institutes of Health); NIH, NCI, Contract Nos. HHSN261201000037C and HHSN261201000032C, Surveillance, Epidemiology and End Results (SEER) Program; The Queen's Medical Center Translational Seed Grant Program; Masami Horio Family Grant.

\section{Footnote}

Reporting Checklist: The authors have completed the MDAR reporting checklist. Available at http://dx. doi. org/10. 21037/gist-20-2

Conflicts of Interest: All authors have completed the ICMJE uniform disclosure form (available at http://dx. doi. org/10. 21037/gist-20-2). Dr. CFL reports grants from National Cancer Institute, during the conduct of the study. The other authors have no conflicts of interest to declare.

Ethical Statement: The authors are accountable for all aspects of the work in ensuring that questions related to the accuracy or integrity of any part of the work are appropriately investigated and resolved. Approval for this research was granted by the Western Institutional Review Board (protocol No. 1168906) and University of Hawaii Human Subjects Committee (2014-302). This study conformed to the provisions of the Declaration of Helsinki (as revised in 2013). Due to the retrospective nature of the data and biospecimen collection, and de-identification of all personal health information, individual consent for this retrospective analysis was waived.

Open Access Statement: This is an Open Access article distributed in accordance with the Creative Commons Attribution-NonCommercial-NoDerivs 4.0 International License (CC BY-NC-ND 4.0), which permits the noncommercial replication and distribution of the article with the strict proviso that no changes or edits are made and the original work is properly cited (including links to both the formal publication through the relevant DOI and the license). See: https://creativecommons.org/licenses/by-nc-nd/4.0/.

\section{References}

1. Kindblom LG, Remotti HE, Aldenborg F, et al. Gastrointestinal pacemaker cell tumor (GIPACT): gastrointestinal stromal tumors show phenotypic characteristics of the interstitial cells of Cajal. Am J Pathol 1998;152:1259-69.

2. Hirota S, Isozaki K, Moriyama $Y$, et al. Gain-of-function mutations of c-kit in human gastrointestinal stromal tumors. Science 1998;279:577-80.

3. Kitamura Y, Hirota S, Nishida T. Molecular pathology of c-kit proto-oncogene and development of gastrointestinal stromal tumors. Ann Chir Gynaecol 1998;87:282-6.

4. Fletcher CD, Berman JJ, Corless C, et al. Diagnosis of gastrointestinal stromal tumors: a consensus approach. Hum Pathol 2002;33:459-65.

5. West RB, Corless CL, Chen X, et al. The novel marker, DOG1, is expressed ubiquitously in gastrointestinal stromal tumors irrespective of KIT or PDGFRA mutation status. Am J Pathol 2004;165:107-13.

6. Wong NA, Shelley-Fraser G. Specificity of DOG1 (K9 clone) and protein kinase $\mathrm{C}$ theta (clone 27) as immunohistochemical markers of gastrointestinal stromal tumour. Histopathology 2010;57:250-8.

7. Novelli M, Rossi S, Rodriguez-Justo M, et al. DOG1 and CD117 are the antibodies of choice in the diagnosis of gastrointestinal stromal tumours. Histopathology 2010;57:259-70.

8. Duensing A, Medeiros F, McConarty B, et al. Mechanisms of oncogenic KIT signal transduction in primary gastrointestinal stromal tumors (GISTs). Oncogene 2004;23:3999-4006.

9. Rossi S, Gasparotto D, Toffolatti L, et al. Molecular and clinicopathologic characterization of gastrointestinal 
stromal tumors (GISTs) of small size. Am J Surg Pathol 2010;34:1480-91.

10. Rubin JL, Sanon M, Taylor DC, et al. Epidemiology, survival, and costs of localized gastrointestinal stromal tumors. Int J Gen Med 2011;4:121-30.

11. Tran T, Davila JA, El-Serag HB. The epidemiology of malignant gastrointestinal stromal tumors: an analysis of 1,458 cases from 1992 to 2000. Am J Gastroenterol 2005;100:162-8.

12. Census 2000 Gateway. 2000. Available online: http://www. census.gov/main/www/cen2000.html

13. Steinau M, Patel SS, Unger ER. Efficient DNA extraction for HPV genotyping in formalin-fixed, paraffin-embedded tissues. J Mol Diagn 2011;13:377-81.

14. Sicinschi LA, Correa P, Bravo LE, et al. Non-invasive genotyping of Helicobacter pylori cagA, vacA, and hopQ from asymptomatic children. Helicobacter 2012;17:96-106.

15. Rota CA, Pereira-Lima JC, Blaya C, et al. Consensus and variable region PCR analysis of Helicobacter pylori 3' region of cagA gene in isolates from individuals with or without peptic ulcer. J Clin Microbiol 2001;39:606-12.

16. Koehler CI, Mues MB, Dienes HP, et al. Helicobacter pylori genotyping in gastric adenocarcinoma and MALT lymphoma by multiplex PCR analyses of paraffin wax embedded tissues. Mol Pathol 2003;56:36-42.

17. Clayton CL, Kleanthous H, Coates PJ, et al. Sensitive detection of Helicobacter pylori by using polymerase chain reaction. J Clin Microbiol 1992;30:192-200.

18. Nomura A, Stemmermann GN, Chyou PH, et al. Helicobacter pylori infection and gastric carcinoma among Japanese Americans in Hawaii. N Engl J Med 1991;325:1132-6.

19. Yamamoto D, Hamada Y, Tsubota Y, et al. Simultaneous development of adenocarcinoma and gastrointestinal stromal tumor (GIST) in the stomach: case report. World J Surg Oncol 2012;10:6.

20. Cai R, Ren G, Wang DB. Synchronous adenocarcinoma and gastrointestinal stromal tumors in the stomach. World J Gastroenterol 2013;19:3117-23.

21. Abraham SC, Krasinskas AM, Hofstetter WL, et al. "Seedling" mesenchymal tumors (gastrointestinal stromal tumors and leiomyomas) are common incidental tumors of the esophagogastric junction. Am J Surg Pathol 2007;31:1629-35.

22. Chan CH, Cools-Lartigue J, Marcus VA, et al. The impact of incidental gastrointestinal stromal tumours on patients undergoing resection of upper gastrointestinal neoplasms.
Can J Surg 2012;55:366-70.

23. Yan Y, Li Z, Liu Y, et al. Coexistence of gastrointestinal stromal tumors and gastric adenocarcinomas. Tumour Biol 2013;34:919-27.

24. Parkin DM, Muir CS. Cancer incidence in five continents. Comparability and quality of data. IARC Sci Publ 1992:45-173.

25. Salam I, Durai D, Murphy JK, et al. Regression of primary high-grade gastric B-cell lymphoma following Helicobacter pylori eradication. Eur J Gastroenterol Hepatol 2001;13:1375-8.

26. IARC Helicobacter pylori Working Group. Helicobacter pylori Eradication as a Strategy for Preventing Gastric Cancer: IARC Working Group Report Volume 8. Lyon: International Agency for Research on Cancer, 2014. Available online: https://publications.iarc.fr/Book-AndReport-Series/Iarc-Working-Group-Reports/-EmHelicobacter-Pylori-Em-Eradication-As-A-Strategy-ForPreventing-Gastric-Cancer-2014

27. Raza MA, Shahab AS, Mazzara P. Gastrointestinal stromal tumors: association with Helicobacter pylori gastritis. Am J Clin Pathol 2012;138:A351.

28. Kiss S, Zsikla V, Frank A, et al. Helicobacter-negative gastritis: polymerase chain reaction for Helicobacter DNA is a valuable tool to elucidate the diagnosis. Aliment Pharmacol Ther 2016;43:924-32.

29. Ramírez-Lázaro MJ, Lario S, Casalots A, et al. Real-time PCR improves Helicobacter pylori detection in patients with peptic ulcer bleeding. PLoS One 2011;6:e20009.

30. Kobayashi M, Yamamoto K, Ogiwara N, et al. Helicobacter heilmannii-like organism in parietal cells: A diagnostic pitfall. Pathol Int 2016;66:120-2.

31. Hole DJ, Quigley EM, Gillis CR, et al. Peptic ulcer and cancer: an examination of the relationship between chronic peptic ulcer and gastric carcinoma. Scand J Gastroenterol 1987;22:17-23.

32. Hansson LE, Nyren O, Hsing AW, et al. The risk of stomach cancer in patients with gastric or duodenal ulcer disease. N Engl J Med 1996;335:242-9.

33. Lee S, Iida M, Yao T, et al. Risk of gastric cancer in patients with non-surgically treated peptic ulcer. Scand J Gastroenterol 1990;25:1223-6.

34. Molloy RM, Sonnenberg A. Relation between gastric cancer and previous peptic ulcer disease. Gut 1997;40:247-52.

35. Keenan JI, Beaugie CR, Jasmann B, et al. Helicobacter species in the human colon. Colorectal Dis 2010;12:48-53.

36. Oliveira AG, das Graças Pimenta Sanna M, Rocha GA, et 
al. Helicobacter species in the intestinal mucosa of patients with ulcerative colitis. J Clin Microbiol 2004;42:384-6.

37. Oh JK, Weiderpass E. Infection and cancer: global distribution and burden of diseases. Ann Glob Health

doi: 10.21037 /gist-20-2

Cite this article as: Kagihara J, Matsuda B, Young KL, Li X, Lao X, Deshpande GA, Omata F, Burnett T, Lynch CF, Hernandez BY, Kuwada SK. Novel association between Helicobacter pylori infection and gastrointestinal stromal tumors (GIST) in a multi-ethnic population. Gastrointest Stromal Tumor 2020;3:1.
2014;80:384-92.

38. Franco MC, Schulz RT, Maluf-Filho F. Opinion: how to manage subepithelial lesions of the upper gastrointestinal tract? World J Gastrointest Endosc 2015;7:1262-7. 
Table S1 GIST cases used for $H$. pylori detection

\begin{tabular}{|c|c|c|c|c|c|c|c|c|}
\hline Sample & Gender & Ethnicity & Age & Place of birth & Tumor site & Tumor class & Tumor grade & Stage \\
\hline GIST 1 & Male & Filipino & $60-69$ & Asia NOS & Gastric antrum & Malignant primary & 2 & Localized \\
\hline GIST 2 & Female & Japanese & 70-79 & Hawaii & Lesser curvature of stomach, NOS & Malignant primary & 1 & Regional, extensive \\
\hline GIST 3 & Male & White & $60-69$ & USA (not Hawaii) & Gastric antrum & Malignant primary & 2 & Localized \\
\hline GIST 4 & Male & Pac Island excl Hawaii & $60-69$ & Pacific Islands & Fundus of stomach & Malignant primary & 1 & Localized \\
\hline GIST 5 & Female & Other & $21-49$ & Hawaii & Small intestine, NOS & Malignant primary & 1 & Localized \\
\hline GIST 6 & Male & Hawaiian & $21-49$ & Hawaii & Overlapping lesion of stomach & Malignant primary & 3 & Regional, extensive \\
\hline GIST 7 & Male & Japanese & $60-69$ & Hawaii & Stomach, NOS & Malignant primary & Unknown, not stated, or N/A & Localized \\
\hline GIST 8 & Female & Filipino & $60-69$ & Asia NOS & Duodenum & Malignant primary & 1 & Localized \\
\hline GIST 9 & Female & Japanese & 70-79 & Hawaii & Colon, NOS & Malignant primary & Unknown, not stated, or N/A & Regional, extensive \\
\hline GIST 10 & Male & Japanese & $21-49$ & USA (not Hawaii) & Duodenum & Malignant primary & 1 & Regional, extensive \\
\hline GIST 11 & Female & Filipino & $50-59$ & Asia NOS & Gastric antrum & Malignant primary & Unknown, not stated, or N/A & Localized \\
\hline GIST 12 & Female & Chinese & $21-49$ & Unknown, not stated, or N/A & Ileum & Malignant primary & 1 & Distant \\
\hline GIST 13 & Female & Chinese & $>80$ & Hawaii & Greater curvature of stomach, NOS & Malignant primary & Unknown, not stated, or N/A & Distant \\
\hline GIST 14 & Male & Chinese & $70-79$ & Hawaii & Duodenum & Malignant primary & Unknown, not stated, or N/A & Localized \\
\hline GIST 15 & Female & Japanese & $60-69$ & Hawaii & Overlapping lesion of small intestine & Malignant primary & 1 & Localized \\
\hline GIST 16 & Female & Hawaiian & $50-59$ & Hawaii & Small intestine, NOS & Malignant primary & 3 & Distant \\
\hline GIST 17 & Male & Hawaiian & $21-49$ & Hawaii & Small intestine, NOS & Malignant primary & Unknown, not stated, or N/A & Distant \\
\hline GIST 18 & Male & Chinese & 70-79 & Hawaii & Overlapping lesion of stomach & Malignant primary & Unknown, not stated, or N/A & Regional, extensive, lymph nodes \\
\hline GIST 19 & Male & Filipino & $50-59$ & Asia NOS & Greater curvature of stomach, NOS & Malignant primary & Unknown, not stated, or N/A & Localized \\
\hline GIST 20 & Female & Korean & 70-79 & Asia NOS & Duodenum & Malignant primary & 4 & Regional, extensive \\
\hline GIST 21 & Female & Japanese & 70-79 & Hawaii & Overlapping lesion of stomach & Malignant primary & 4 & Localized \\
\hline GIST 22 & Female & Korean & $50-59$ & Unknown, not stated, or N/A & Overlapping lesion of stomach & Malignant primary & Unknown, not stated, or N/A & Localized \\
\hline GIST 23 & Male & Filipino & $>80$ & Asia NOS & lleum & Malignant primary & Unknown, not stated, or N/A & Distant \\
\hline GIST 24 & Female & Chinese & 70-79 & Hawaii & lleum & Malignant primary & 1 & Localized \\
\hline GIST 25 & Female & Japanese & $>80$ & Hawaii & Duodenum & Malignant primary & 4 & Localized \\
\hline GIST 26 & Male & Japanese & 70-79 & Unknown, not stated, or N/A & Stomach, NOS & Malignant primary & Unknown, not stated, or N/A & Unknown, not stated, or N/A \\
\hline GIST 27 & Male & White & $50-59$ & Unknown, not stated, or N/A & Stomach, NOS & Malignant primary & Unknown, not stated, or N/A & Unknown, not stated, or N/A \\
\hline GIST 28 & Male & Filipino & $60-69$ & Unknown, not stated, or N/A & Rectosigmoid junction & Malignant primary & Unknown, not stated, or N/A & Unknown, not stated, or N/A \\
\hline GIST 29 & Female & Japanese & $>80$ & Unknown, not stated, or N/A & Stomach, NOS & Malignant primary & Unknown, not stated, or N/A & Unknown, not stated, or N/A \\
\hline GIST 30 & Male & Chinese & $21-49$ & Unknown, not stated, or N/A & Stomach, NOS & Malignant primary & Unknown, not stated, or N/A & Unknown, not stated, or N/A \\
\hline GIST 31 & Female & Hawaiian & $60-69$ & Unknown, not stated, or N/A & Stomach, NOS & Malignant primary & Unknown, not stated, or N/A & Unknown, not stated, or N/A \\
\hline GIST 32 & Female & Japanese & 70-79 & Unknown, not stated, or N/A & Stomach, NOS & Malignant primary & Unknown, not stated, or N/A & Unknown, not stated, or N/A \\
\hline GIST 33 & Female & Japanese & $>80$ & Unknown, not stated, or N/A & Stomach, NOS & Malignant primary & Unknown, not stated, or N/A & Unknown, not stated, or N/A \\
\hline GIST 34 & Male & Japanese & $60-69$ & Unknown, not stated, or N/A & Small intestine, NOS & Malignant primary & 1 & Unknown, not stated, or N/A \\
\hline GIST 35 & Female & Chinese & $60-69$ & Unknown, not stated, or N/A & Small intestine, NOS & Malignant primary & 1 & Unknown, not stated, or N/A \\
\hline GIST 36 & Female & Japanese & $50-59$ & Unknown, not stated, or N/A & Small intestine, NOS & Malignant primary & 1 & Unknown, not stated, or N/A \\
\hline GIST 37 & Female & Japanese & $>80$ & Unknown, not stated, or N/A & Stomach, NOS & Malignant primary & 1 & Unknown, not stated, or N/A \\
\hline GIST 38 & Female $^{*}$ & Hawaiian & $50-59$ & Unknown, not stated, or N/A & Cardia, esophagogastric junction & Malignant primary & 1 & Unknown, not stated, or N/A \\
\hline GIST 39 & Female & Japanese & $50-59$ & Unknown, not stated, or N/A & Stomach, NOS & Malignant primary & 1 & Unknown, not stated, or N/A \\
\hline GIST 40 & Male & Japanese & $70-79$ & Unknown, not stated, or N/A & Stomach, NOS & Malignant primary & 1 & Unknown, not stated, or N/A \\
\hline GIST 41 & Male & Hawaiian & $21-49$ & Unknown, not stated, or N/A & Stomach, NOS & Malignant primary & 1 & Unknown, not stated, or N/A \\
\hline GIST 42 & Male & Filipino & $>80$ & Unknown, not stated, or N/A & Stomach, NOS & Malignant primary & 1 & Unknown, not stated, or N/A \\
\hline GIST 43 & Female $^{*}$ & Hawaiian & $70-79$ & Unknown, not stated, or N/A & Stomach, NOS & Malignant primary & 1 & Unknown, not stated, or N/A \\
\hline GIST 44 & Male & White & $60-69$ & Unknown, not stated, or N/A & Stomach, NOS & Malignant primary & 1 & Unknown, not stated, or N/A \\
\hline GIST 45 & Male & Pac Island excl Hawaii & $21-49$ & Unknown, not stated, or N/A & Stomach, NOS & Malignant primary & 1 & Unknown, not stated, or N/A \\
\hline GIST 46 & Female & Pac Island excl Hawaii & $21-49$ & Unknown, not stated, or N/A & Stomach, NOS & Malignant primary & 1 & Unknown, not stated, or N/A \\
\hline GIST 47 & Female & White & $50-59$ & Unknown, not stated, or N/A & Stomach, NOS & Malignant primary & 1 & Unknown, not stated, or N/A \\
\hline GIST 48 & Male & White & 70-79 & Unknown, not stated, or N/A & Stomach, NOS & Malignant primary & 1 & Unknown, not stated, or N/A \\
\hline GIST 49 & Male $^{* *}$ & Japanese & $70-70$ & Unknown, not stated, or N/A & Stomach, NOS & Malignant primary & 1 & Unknown, not stated, or N/A \\
\hline GIST 50 & Female & Japanese & $>80$ & Unknown, not stated, or N/A & Stomach, NOS & Malignant primary & 2 & Unknown, not stated, or N/A \\
\hline GIST 51 & Female & Japanese & $>80$ & Unknown, not stated, or N/A & Stomach, NOS & Malignant primary & 2 & Unknown, not stated, or N/A \\
\hline GIST 52 & Male & Hawaiian & $70-79$ & Unknown, not stated, or N/A & Stomach, NOS & Malignant primary & 3 & Unknown, not stated, or N/A \\
\hline GIST 53 & Male & Hawaiian & $50-59$ & Unknown, not stated, or N/A & Small intestine, NOS & Malignant primary & 3 & Unknown, not stated, or N/A \\
\hline GIST 54 & Male & Pac Island excl Hawaii & $60-69$ & Unknown, not stated, or N/A & Stomach, NOS & Malignant primary & 3 & Unknown, not stated, or N/A \\
\hline GIST 55 & Male & White & $50-59$ & Unknown, not stated, or N/A & $\begin{array}{l}\text { Specified parts of peritoneum } \\
\text { (including omentum and mesentery) }\end{array}$ & Malignant primary & 3 & Unknown, not stated, or N/A \\
\hline GIST 56 & Male & Japanese & $50-59$ & Unknown, not stated, or N/A & Stomach, NOS & Malignant primary & 3 & Unknown, not stated, or N/A \\
\hline GIST 57 & Male & Pac Island excl Hawaii & $21-49$ & Unknown, not stated, or N/A & Stomach, NOS & Malignant primary & 4 & Unknown, not stated, or N/A \\
\hline GIST 58 & Female & Chinese & $60-69$ & Unknown, not stated, or N/A & Stomach, NOS & Malignant primary & $4^{* * *}$ & Unknown, not stated, or N/A \\
\hline GIST 59 & Female & Korean & 70-79 & Unknown, not stated, or N/A & Liver & Malignant primary & $4^{* * *}$ & Unknown, not stated, or N/A \\
\hline GIST 60 & Male & Chinese & $50-59$ & Unknown, not stated, or N/A & Rectum, NOS & Malignant primary & Unknown, not stated, or N/A & Unknown, not stated, or N/A \\
\hline GIST 61 & Male & Japanese & $60-69$ & Unknown, not stated, or N/A & Anus, NOS & Malignant primary & Unknown, not stated, or N/A & Unknown, not stated, or N/A \\
\hline GIST 62 & Male & Filipino & $70-79$ & Unknown, not stated, or N/A & Stomach, NOS & Malignant primary & Unknown, not stated, or N/A & Unknown, not stated, or N/A \\
\hline GIST 63 & Female & Chinese & 70-79 & Unknown, not stated, or N/A & Stomach, NOS & Malignant primary & 1 & Unknown, not stated, or N/A \\
\hline GIST 64 & Female & White & 82 & lowa & Overlapping lesion of stomach & Malignant primary & 2 & Localized \\
\hline GIST 65 & Male & White & 48 & lowa & Lesser curvature of stomach, NOS & Malignant primary & 1 & Localized \\
\hline GIST 66 & Female & White & 72 & lowa & Overlapping lesion of stomach & Malignant primary & Unknown, not stated, or N/A & Localized \\
\hline GIST 67 & Female & White & 81 & lowa & Overlapping lesion of stomach & Malignant primary & Unknown, not stated, or N/A & Regional, extensive \\
\hline GIST 68 & Male & White & 70 & lowa & Stomach, NOS & Malignant primary & 4 & Localized \\
\hline GIST 69 & Female & White & 81 & lowa & Cardia, esophagogastric junction & Malignant primary & Unknown, not stated, or N/A & Regional, extensive \\
\hline GIST 70 & Male & White & 72 & lowa & Cardia, esophagogastric junction & Malignant primary & Unknown, not stated, or N/A & Localized \\
\hline GIST 71 & Male & White & 72 & lowa & Cardia, esophagogastric junction & Malignant primary & Unknown, not stated, or N/A & Localized \\
\hline
\end{tabular}

*, Specimen with concurrent gastric adenocarcinoma; ${ }^{* \star}$, concurrent diagnosis of pancreatic well-differentiated neuroendocrine tumor; ${ }^{* * *}$, liver metastasis. H. pylori, Helicobacter pylori; GIST, gastrointestinal stromal tumors. 


\begin{tabular}{|c|c|c|c|c|c|c|c|c|c|c|c|c|c|}
\hline \multicolumn{7}{|c|}{ GIST cases } & \multicolumn{7}{|c|}{ Controls } \\
\hline H. pylori gene & bgl control & HP1177 & $16 S$ rRNA & iceA & ureB & $\operatorname{vac} A$ & H. pylori gene & bgl control & HP1177 & $16 S$ rRNA & $i c e A$ & ureB & $\operatorname{vac} A$ \\
\hline Blank control & - & - & - & - & - & - & Blank control & - & - & - & - & - & - \\
\hline Negative control & - & - & - & - & - & - & Negative control & - & - & - & - & - & - \\
\hline Positive control & + & + & + & + & + & + & Positive control & + & + & + & + & + & + \\
\hline GIST 1 & + & & + & & & & Control 1 & + & & & + & & \\
\hline GIST 2 & + & & & & & & Control 2 & + & & & & & \\
\hline GIST 3 & + & & & & & & Control 3 & + & & & & & \\
\hline GIST 4 & + & & & + & & & Control 4 & + & & & & & \\
\hline GIST 5 & + & & & & + & & Control 5 & + & & & + & & \\
\hline GIST 6 & + & & + & + & + & + & Control 6 & + & & & & + & + \\
\hline GIST 7 & + & & & & & & Control 7 & + & & & & & \\
\hline GIST 8 & + & & & & & & Control 8 & + & & & & & \\
\hline GIST 9 & + & & & + & & & Control 9 & + & & & & & \\
\hline GIST 10 & + & & & & & & Control 10 & + & & & & & \\
\hline GIST 11 & + & & & & & & Control 11 & + & & & & & \\
\hline GIST 12 & + & & + & + & & & Control 12 & + & & & & & \\
\hline GIST 13 & + & & & + & & & Control 13 & + & + & & & & + \\
\hline GIST 14 & + & & & & & & Control 14 & + & & & & & \\
\hline GIST 15 & + & & + & + & & & Control 15 & + & & & & & \\
\hline GIST 16 & + & & + & + & & & Control 16 & + & & & & & \\
\hline GIST 17 & + & & + & & & & Control 17 & + & & & + & & \\
\hline GIST 18 & + & & + & + & & & Control 18 & + & & & & & \\
\hline GIST 19 & + & + & + & + & & + & Control 19 & + & & & & & \\
\hline GIST 20 & + & & + & & & & Control 20 & + & & & & & \\
\hline GIST 21 & + & & & & & & Control 21 & + & & & & & \\
\hline GIST 22 & + & & & & & & Control 22 & + & & & & & \\
\hline GIST 23 & + & & + & + & & + & Control 23 & + & & & & & \\
\hline GIST 24 & + & & & & + & & Control 24 & + & & & + & & \\
\hline GIST 25 & + & & + & + & + & + & Control 25 & + & & & & & \\
\hline GIST 26 & + & & & & & & Control 26 & + & & & + & + & \\
\hline GIST 27 & + & & & + & & & Control 27 & + & & & & & \\
\hline GIST 28 & + & & & & & & Control 28 & + & & & & & \\
\hline GIST 29 & + & & + & & + & & Control 29 & + & & & & & \\
\hline GIST 30 & + & & + & & + & + & Control 30 & + & & & & & \\
\hline GIST 31 & + & & + & & & + & Control 31 & + & & & + & & \\
\hline GIST 32 & + & + & + & & + & & Control 32 & + & & & & & \\
\hline GIST 33 & + & & & & + & + & Control 33 & + & & & + & & \\
\hline GIST 34 & + & + & & & & & Control 34 & + & & & + & & \\
\hline GIST 35 & + & & & & & & Control 35 & + & & & & & \\
\hline GIST 36 & + & & & & & & Control 36 & + & & & + & + & \\
\hline GIST 37 & + & & & + & & & Control 37 & + & & & & & \\
\hline GIST 38 & + & & & & & & Control 38 & + & & & & & \\
\hline GIST 39 & + & & & & & & Control 39 & + & & & & & \\
\hline GIST 40 & + & & & & & & Control 40 & + & & & & & \\
\hline GIST 41 & + & & & & & + & Control 41 & + & & + & & + & + \\
\hline GIST 42 & + & & & & + & & Control 42 & + & & & & & \\
\hline GIST 43 & + & & & & & & Control 43 & + & & & & & \\
\hline GIST 44 & + & + & & & + & & Control 44 & + & & & & & \\
\hline GIST 45 & + & + & & & & & Control 45 & + & & & & & \\
\hline GIST 46 & + & + & + & + & + & + & Control 46 & + & & + & & + & + \\
\hline GIST 47 & + & + & + & & & & Control 47 & + & & & & & \\
\hline GIST 48 & + & + & & & & & Control 48 & + & & & & & \\
\hline GIST 49 & + & + & & & & & Control 49 & + & & & & & \\
\hline GIST 50 & + & & & & & & Control 50 & + & & & & & \\
\hline GIST 51 & + & & & & & & Control 51 & + & & & & & \\
\hline GIST 52 & + & + & & + & & & Control 52 & + & & & & & \\
\hline GIST 53 & + & & & & & & Control 53 & + & & & & & \\
\hline GIST 54 & + & & & & & & Control 54 & + & & & & & \\
\hline GIST 55 & + & + & & & & & Control 55 & + & & & & & \\
\hline GIST 56 & + & + & & & & & Control 56 & + & & & & & \\
\hline GIST 57 & + & + & & & & & Control 57 & + & & & & & \\
\hline GIST 58 & + & + & & & & & Control 58 & + & & & & & \\
\hline GIST 59 & + & & + & & & + & Control 59 & + & & & & & \\
\hline GIST 60 & + & + & & & & & Control 60 & + & & & & & \\
\hline GIST 61 & + & & & & & + & Control 61 & + & & & & & \\
\hline GIST 62 & + & + & & & & & Control 62 & + & & & & & \\
\hline GIST 63 & + & + & & & & & Control 63 & + & & & & & \\
\hline GIST 64 & + & + & & + & + & + & Control 64 & + & & & & & \\
\hline GIST 65 & + & + & & + & & + & Control 65 & + & & & & & \\
\hline GIST 66 & + & & + & + & + & & Total & 65 & 1 & 2 & 5 & 9 & 4 \\
\hline GIST 67 & + & + & & + & + & & & & & & & & \\
\hline GIST 68 & + & & & & & & & & & & & & \\
\hline GIST 69 & + & & & & & & & & & & & & \\
\hline GIST 70 & + & & & & & & & & & & & & \\
\hline GIST 71 & + & + & & + & & + & & & & & & & \\
\hline Total & 71 & 21 & 18 & 25 & 15 & 14 & & & & & & & \\
\hline
\end{tabular}

H. pylori, Helicobacter pylori; GIST, gastrointestinal stromal tumors. 